Research Article

\title{
Sticking Fault Detecting Method for CARIMA Model
}

\author{
Toyoaki Tanikawa ${ }^{1}$, Henmi Tomohiro ${ }^{2,}$ \\ ${ }^{1}$ Advanced Course in Industrial and Systems Engineering, National Institute of Technology, Kagawa College, Takamatsu, Kagawa 761-8058, Japan \\ ${ }^{2}$ Department of Electrical and Computer Engineering, National Institute of Technology, Kagawa College, Takamatsu, Kagawa 761-8058, Japan
}

\section{ARTICLE INFO}

Article History

Received 10 August 2018

Accepted 19 October 2018

Keywords

Fault detection

sticking fault

CARIMA model

\begin{abstract}
This paper proposes a sticking fault detecting method for controlled auto-regressive integrated moving average model (CARIMA) which detect the sticking fault of control input and feedback signal. It consists of model estimation using recursive least square method with the forgetting factor and fault detection. In the fault detection, an evaluation function is introduced, and it generates a fault signal from the input and output data. Numerical simulations are performed, and it is shown that this method can detect the sticking fault.
\end{abstract}

(C) 2018 The Authors. Published by Atlantis Press SARL. This is an open access article under the CC BY-NC license (http://creativecommons.org/licenses/by-nc/4.0/).

\section{INTRODUCTION}

If a system with a fault continues to be operated, it can cause a serious accident or a considerable damage. Thus, it is important to detect faults and compensate them, and many fault detection methods have been proposed [1,2]. The advantage of fault detection is that the safety is improved, you can cope with the fault more promptly, and sometimes the system can be controlled compensating the fault. There are two kinds in fault detection, signal-based detection and model-based one. The signal-based detection is for example a method using spectral analysis, statistical signal analysis or pattern recognition, while the modelbased detection uses an observer or a parameter estimation [3]. In model-based detection, a general method detecting additive faults is proposed by Isermann [4].

In detecting a fault where control input or feedback signal is fixed, a general detection method is not established. In this paper, we propose a model-based detection method for the sticking fault of control input and feedback signal on a system expressed as a controlled auto-regressive integrated moving average (CARIMA) model. Then, we discuss its effectiveness performing a numerical simulation.

\section{STICKING FAULT DETECTING METHOD}

In this section, we discuss how to detect sticking fault of control input and feedback signal on the general linear systems.

\footnotetext{
*Corresponding author.Emails: toyoakipd0@gmail.com; henmi@t.kagawa-nct.ac.jp; www.kagawa-nct.ac.jp
}

\subsection{Problem Statement}

First, we assume the control object is single input and single output system and expressed in Eq. (1) as following CARIMA model:

$$
\begin{aligned}
A\left[z^{-1}\right] y_{m}(k) & =z^{-k_{m}} B\left[z^{-1}\right] u_{m}(k)+C\left[z^{-1}\right] \frac{\xi(k)}{\Delta} \\
A\left[z^{-1}\right] & =1+a_{1} z^{-1}+a_{2} z^{-2}+\ldots+a_{n} z^{-n} \\
B\left[z^{-1}\right] & =b_{0}+b_{1} z^{-1}+b_{2} z^{-2}+\ldots+b_{m} z^{-m} \\
C\left[z^{-1}\right] & =1+c_{1} z^{-1}+c_{2} z^{-2}+\cdots+c_{l} z^{-l}
\end{aligned}
$$

where $y_{m}(k)$ represents a system output, $u_{m}(k)$ is a control input, $\xi(k)$ is a white Gaussian noise with 0 mean and $k_{m}$ is a time delay. $z^{-1}$ is a backshift operator where $z^{-1} y_{m}(k)=y_{m}(k-1)$ and $\Delta$ is a difference operator defined as $\Delta=1-z^{-1}$. System parameters, $a, b$ and $c$ in Eq. (1) changes gradually or do not change at all. We suppose the reference value of the control system changes depending on time.

Let us define the sticking fault. When the sticking fault of control input occurs, the input to the system $u_{m}(k)$ becomes a white noise and expressed in Eq. (2):

$$
u_{m}(k)= \begin{cases}u(k) & \text { (normal) } \\ u_{f}+\xi_{u}(k) & \text { (fault) }\end{cases}
$$

where $u(k)$ is a control input calculated by the controller, $u_{f}$ is a constant value and $\xi_{u}(k)$ is a white Gaussian noise with 0 mean. When the sticking fault of feedback signal occurs, the feedback signal to the controller $y(k)$ becomes a white noise and expressed in Eq. (3):

$$
y(k)= \begin{cases}y_{m}(k) & \text { (normal) } \\ y_{f}+\xi_{y}(k) & (\text { fault })\end{cases}
$$


where $y_{f}$ is a constant value and $\xi_{y}(k)$ is a white Gaussian noise with 0 mean.

Figure 1 shows a block diagram of the fault detection for the above-mentioned faults. This method consists of two parts, model estimation and fault analysis.

\subsection{Model Estimation}

Least square method with the forgetting factor [5] is used for the model estimation. In this method, the cost function is given in Eq. (4):

$$
I_{N}(\boldsymbol{\theta})=\sum_{k=1}^{N} \lambda^{N-k}[y(k)-\hat{y}(k \mid \boldsymbol{\theta})]^{2}
$$

where $\theta$ is a system parameter vector, $\lambda$ is a forgetting factor where $0<\lambda \leq 1, \hat{y}(k \mid \boldsymbol{\theta})$ is an estimated output and $N$ is the number of the input-output data. The estimated parameter $\hat{\boldsymbol{\theta}}$ which minimizes the cost function on CARIMA model is obtained by the following recursive algorithm [Eq. (5)]

$$
\begin{aligned}
& \hat{\boldsymbol{\theta}}(k)=\hat{\boldsymbol{\theta}}(k-1)+\frac{\boldsymbol{P}(k-1) \psi(k)}{\lambda+\psi^{T}(k) \boldsymbol{P}(k-1) \psi(k)} \in(k) \\
& \boldsymbol{P}(k)=\frac{1}{\lambda}\left[\boldsymbol{P}(k-1)-\frac{\boldsymbol{P}(k-1) \psi(k) \psi^{T}(k) \boldsymbol{P}(k-1)}{\lambda+\psi^{T}(k) \boldsymbol{P}(k-1) \psi(k)}\right] \\
& \epsilon(k)=\Delta y(k)-\psi^{T}(k) \hat{\boldsymbol{\theta}}(k-1) \\
& \eta(k)=\Delta y(k)-\psi^{T}(k) \hat{\theta}(k)
\end{aligned}
$$

$\epsilon(k)$ is a priori error and $\eta(k)$ is a posterior error. The parameter vector $\hat{\theta}(k)$ and the data vector $\psi(k)$ are defined in Eq. (6) as follows:

$$
\begin{aligned}
\hat{\theta}(k)= & {\left[\hat{a}_{1}(k), \cdots, \hat{a}_{n}(k), \hat{b}_{0}(k), \cdots, \hat{b}_{m}(k), \hat{c}_{1}(k), \cdots, \hat{c}_{l}(k)\right]^{T} } \\
\psi(k)= & {\left[-\Delta y(k-1), \cdots,-\Delta y(k-n), \Delta u\left(k-k_{m}\right), \cdots,\right.} \\
& \left.\Delta u\left(k-k_{m}-m\right), \eta(k-1), \cdots, \eta(t-l)\right]^{T}
\end{aligned}
$$

where $\hat{a}, \hat{b}$ and $\hat{c}$ represent the estimated parameter of $a, b$ and $c$ in Eq. (1) respectively. The initial value of the covariance matrix $\boldsymbol{P}(k)$ is given in Eq. (7):

$$
P(0)=\gamma I
$$

where $\boldsymbol{I}$ is an identity matrix. $\gamma$ is usually set as a large value like $10^{3}$ or $10^{4}$.

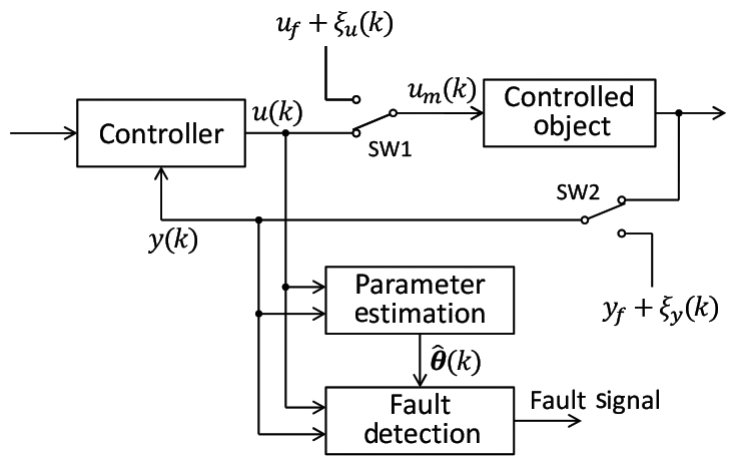

Figure 1 Block diagram of sticking fault detection
The weight of a past input-output data in cost function $I_{N}(\theta)$ becomes smaller as the time goes by due to the forgetting factor. Thus, the estimated parameter $\hat{\boldsymbol{\theta}}(k)$ is calculated using resent data mainly. Thus, this parameter estimation method is also effective for systems where its parameters change.

\subsection{Fault Detection}

Figure 2 shows the outline of the fault detection. To detect sticking fault, it uses the estimated system parameters and the input-output data of past $n_{d}$ steps called detecting period. The fault state is evaluated by the following Eq. (8):

$$
V(k)=\frac{\sum_{i=k-n_{d}+1}^{k}\left\{\Delta \widehat{A}\left[z^{-1}\right] y(i)-z^{-k_{m}} \Delta \widehat{B}\left[z^{-1}\right] u(i)\right\}^{2}}{\sum_{i=k-n_{d}+1}^{k}\left\{\Delta \widehat{A}\left[z^{-1}\right] y(i)\right\}^{2}+\beta}
$$

where $\beta$ is an infinitesimal value to avoid dividing by $0 . \widehat{A}\left[z^{-1}\right]$ and $\widehat{B}\left[z^{-1}\right]$ are the estimated system parameter polynomials at step $k-n_{d}-n_{m}$ which are gained from $\hat{\theta}\left(k-n_{d}-n_{m}\right)$ calculated in Eq. (5), where $n_{m}$ is the order of the system defined by $n_{m}=\max \{m, n\}+1$. It is assumed that $\hat{\theta}\left(k-n_{d}-n_{m}\right)$ is a good estimation of the actual system at step $k$ because we are focusing on systems whose parameters do not change abruptly.

We assume a situation that a fault has occurred. The estimated parameters become useless values after the fault. However, at a moment like Figure 2, estimated parameter $\hat{\theta}\left(k-n_{d}-n_{m}\right)$ is still valid and only the input-output data after the fault are used for the evaluation value $V(k)$. Then $V(k)$ becomes bigger than 1 as being mentioned in Section 3, and a fault signal is generated after satisfying the following inequality [Eq. (9)]:

$$
V(k)>V_{t h}
$$

where $V_{\text {th }}$ is a threshold value set as $V_{\text {th }}>1$. After detecting the fault, it stops updating the estimated parameters in Eq. (5).

\section{STICKING FAULT EVALUATION VALUE}

We discuss the size of the evaluation value $V(k)$ on the normal state and the fault one. First, let us define the error polynomials of the system parameters, $E_{A}\left[z^{-1}\right]$ and $E_{B}\left[z^{-1}\right]$ as Eq. (10):

$$
\begin{aligned}
& E_{A}\left[z^{-1}\right]=\widehat{A}\left[z^{-1}\right]-A\left[z^{-1}\right] \\
& E_{B}\left[z^{-1}\right]=\widehat{B}\left[z^{-1}\right]-B\left[z^{-1}\right]
\end{aligned}
$$

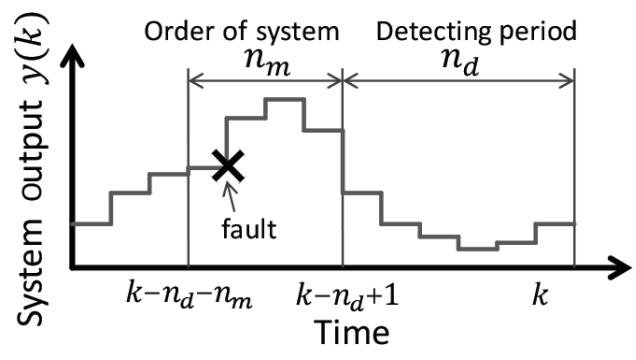

Figure 2 Outline of fault detection 
Using Eqs. (1-3) and (10) and ignoring the infinitesimal value $\beta$, evaluation function in Eq. (8) on the normal state can be deformed as Eq. (11):

$$
V(k)=\frac{\sum_{i}\left\{\Delta E_{A} y(i)-z^{-k_{m}} \Delta E_{B} u(i)+C \xi(i)\right\}^{2}}{\sum_{i}\left\{z^{-k_{m}} \Delta B u(i)+\Delta E_{A} y(i)+C \xi(i)\right\}^{2}}
$$

where the polynomial expression is abbreviated like $B$ instead of $B\left[z^{-1}\right]$, and $\sum_{i}$ means $\sum_{i=k-n_{d}+1}^{k}$. Assuming that the terms of error polynomials and that of white noise $\xi(i)$ are relatively small compared to the term of $u(i)$ in Eq. (11), $V(k) \lesssim 1$ is gained.

On the other hand, after the sticking fault of the input has occurred, the evaluation value at a moment described in Figure 2 is deformed using Eq. (2) of the fault state and Eqs. (1), (3) and (10), and expressed as Eq. (12):

$$
V(k)=\frac{\sum_{i}\left\{-z^{-k_{m}} \Delta \widehat{B}\left(u(i)-\xi_{u}(i)\right)+\Delta E_{A} y(i)+C \xi(i)\right\}^{2}}{\sum_{i}\left\{\Delta E_{A} y(i)+z^{-k_{m}} \Delta B \xi_{u}(i)+C \xi(i)\right\}^{2}}
$$

while the evaluation value on a sticking fault of the feedback signal is deformed using Eq. (3) of the fault state, and expressed as Eq. (13):

$$
V(k)=\frac{\sum_{i}\left\{-z^{-k_{m}} \Delta \widehat{B} u(i)+\Delta \widehat{A} \xi_{y}(i)\right\}^{2}}{\sum_{i}\left\{\Delta \widehat{A} \xi_{y}(i)\right\}^{2}}
$$

Because the terms of error polynomials and white noises are smaller than that of $u(i)$ as assumed before, $V(k) \gtrsim 1$ is obtained in both Eqs. (12) and (13). Therefore, the evaluation value becomes greater after the sticking fault occurs, and the fault can be detected by Eq. (9). However, the threshold $V_{\text {th }}$ has to be set based on an input-output data on an actual system or a simulation results because the evaluation value $V(k)$ is dependent on the estimation error and disturbances.

Systems usually have limitations of the control input. When designing a controller considering the limitation, higher and lower constant limits are generally used. However, Eqs. (11-13) state that $\Delta u(i)$ has to have a value, and $\Delta u(i)$ can be 0 when the input is limited by the constant value. Thus, the limitation has to be dependent on time, and is given by Eq. (14):

$$
\begin{aligned}
u(k) & =\left\{\begin{array}{l}
u_{\text {max }}(k), u_{c}(k)>u_{\text {max }}(k) \\
u_{c}(k), u_{\text {min }}(k) \leq u_{c}(k) \leq u_{\text {max }}(k) \\
u_{\text {min }}(k), u_{c}(k)<u_{\text {min }}(k)
\end{array}\right. \\
u_{\text {max }}(k) & =A_{\text {sin }}(\omega k)+u_{h} \\
u_{\text {min }}(k) & =A_{\text {sin }}(\omega k)+u_{l}
\end{aligned}
$$

where $u_{c}(k)$ is the control input calculated by the controller, and $A, \omega, u_{h}$ and $u_{l}$ are setting parameters.

\section{SIMULATION EXAMPLE}

To verify the effectiveness of this fault detecting method, we apply it to a system expressed as Eq. (15):

$$
\begin{aligned}
& A\left[z^{-1}\right]=1-1.307 z^{-1}+0.3768 z^{-2} \\
& B\left[z^{-1}\right]=0.05158+0.03729 z^{-1}
\end{aligned}
$$

where $k_{m}=1$. We set the disturbance characteristics polynomials $C\left[z^{-1}\right]$ as $C\left[z^{-1}\right]=1$. The system parameter polynomials do not change throughout the simulation. $\xi(k), \xi_{u}(k)$ and $\xi_{y}(k)$ are set as a zero mean white Gaussian noise with the variance of $0.01^{2}, 0.1^{2}$ and $0.005^{2}$ respectively.

In the model estimation, we assume that the order of the system parameter polynomials $l, m$ and $n$ and the time delay $k_{m}$ are known. Then, the estimated parameter vector $\hat{\boldsymbol{\theta}}(k)$ is given in Eq. (16):

$$
\hat{\boldsymbol{\theta}}(k)=\left[\hat{a}_{1}(k), \hat{a}_{2}(k), \hat{b}_{0}(k), \hat{b}_{1}(k)\right]^{T}
$$

We set $\gamma$ to $10^{3}$ and $\lambda$ to 0.98 . In the fault detection, $n_{d}=20, V_{t h}=$ 1.5 and $\beta=10^{-10}$ are used.

Generalized predictive control [6] which uses CARIMA model to predict the output is used as a controller. When designing it, Eq. (14) is used for the internal model, and $N_{1}=1, N_{2}=5, N U=5$, $\lambda(j)=0.01$, and time constant of the reference trajectory $\tau=0.3 \mathrm{~s}$ are taken. For the input limitation, $A=1, \omega=0.1 \pi, u_{h}=4$ and $u_{l}=-4$ are used.

Figure 3 shows a simulation result where the sticking fault of control input occurs at a step 400. $r(k)$ represents reference value and the dashed line on the graph $V(k)$ is the threshold value $V_{t h}$. The evaluate value $V(k)$ is normally smaller than 1 , and it becomes greater after the fault occurs. It surpasses $V_{t h}$ at a step 406, and then the fault signal is generated. $V(k)$ takes relatively great value on the beginning due to the error of the parameter estimation.

Figure 4 shows a result of the sticking fault of feedback signal. Like Figure $3, V(k)$ becomes greater after the fault, and the fault signal is generated at a step 404 .

\section{CONCLUSION}

We proposed the sticking fault detecting method in which the evaluation function is introduced. It evaluates the input-output data on
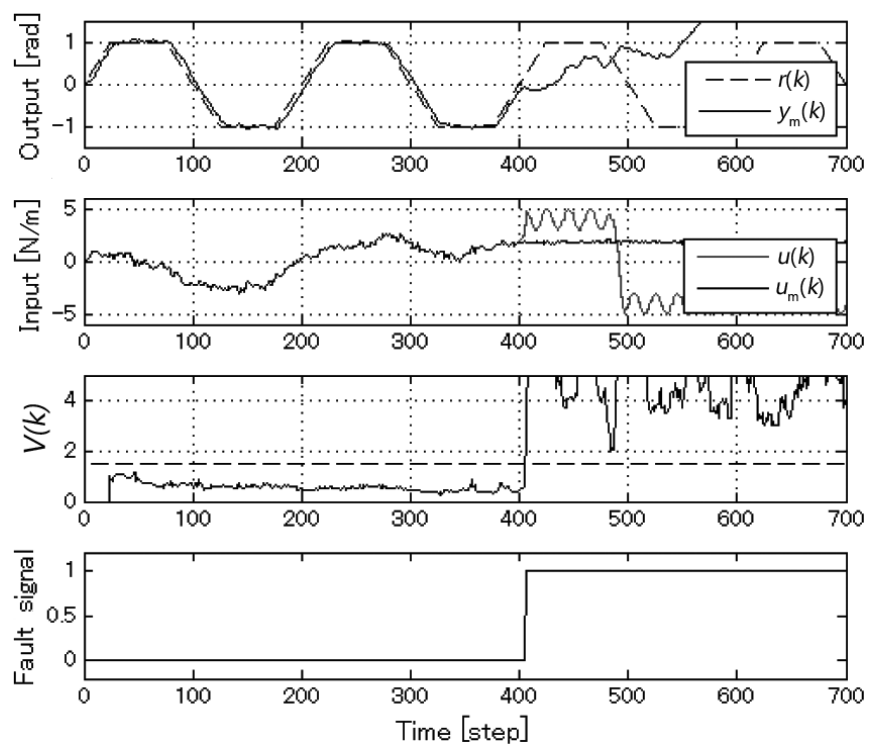

Figure 3 Detection result on control input sticking fault 

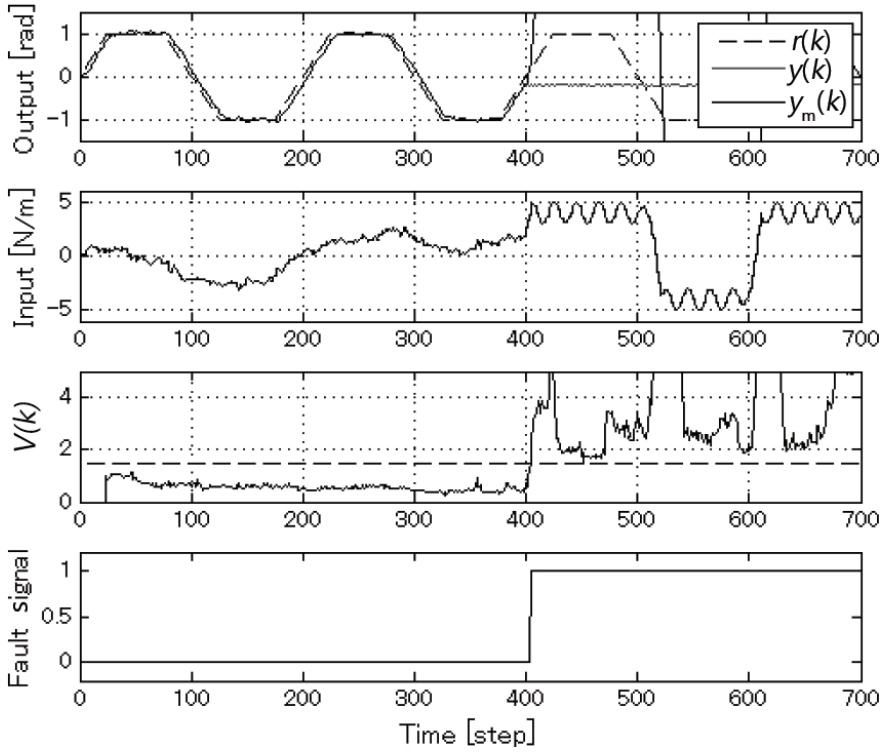

Figure $4 \mid$ Detection result on feedback sticking fault

a detecting period, using the past estimated parameter calculated by the recursive least square method. Performing a numerical simulation, it is confirmed that the sticking fault of control input and feedback signal can be detected.

\section{Authors Introduction}

\section{Mr. Toyoaki Tanikawa}

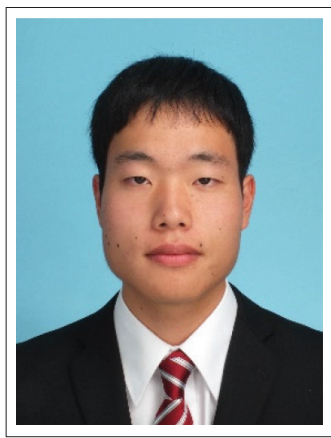

\section{ACKNOWLEDGMENTS}

This work was supported by JSPS KANKENHI (Grant Number 15K21591).

\section{REFERENCES}

[1] M. Takahashi, Self-repairing control utilizing instability by failures, (Performance Assessment and Design of Control Systems), Syst. Control Inform. 58 (2014), 111-116 (in Japanese) (Special issue).

[2] A. Inoue, M. Deng, Y. Shinichi, S. Okazaki, Fault detection to a process control experimental system by using an adaptive observer, Trans. Soc. Inst. Control Eng. 43 (2007), 621-623 (in Japanese).

[3] T. Hukuda, Diagnosis on Machinery (part 1) - the outline and an evaluation of its diagnostic reliability, J. Jpn. Soc. Safety Eng. 36 (1997), 247-252 (in Japanese).

[4] R. Isermann, Model-based Fault-detection and Diagnosis Status and Applications, IFAC Automatic Control in Aerospace, Saint-Petersburg, Russia, 2004, pp. 49-60.

[5] L. Ljung, T. Söderström, Theory and Practice of Recursive Identification, MIT Press, Cambridge, MA, 1983

[6] D.W. Clarke, C. Mohtadi, P.S. Tuffs, Generalized predictive control-part I. The basic algorithm, Automatica 23 (1987), 137-148.

\section{Dr. Tomohiro Henmi}

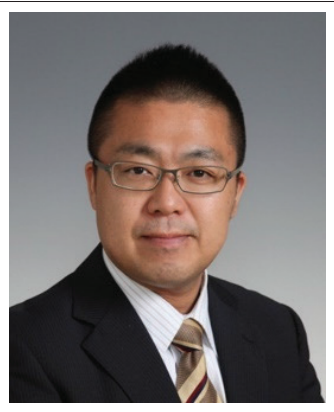

He is an Assistant Professor at the Department of Electro-Mechanical Engineering, National Institute of Technology, Kagawa College, Japan. He received his $\mathrm{PhD}$ from Okayama University, Japan in 2005. His research interests include robotics and control of underactuated non-linear systems. 